\title{
Research on Architecture Design of Urban Comprehensive Management Service Platform
}

\author{
Wang Jichuan, Wang Shengjin* \\ Shanghai Urban Construction Vocational College, Shanghai, 201415, China
}

\begin{abstract}
Information platform can connect all the urban management links and it is a key tool to improve the efficiency and quality of urban comprehensive management. In recent years, the construction of the urban management information platform has progressed rapidly, but problems such as insufficient resource sharing within the platform, poor information communication between departments, active supervision and monitoring are still outstanding and difficult to adapt to the city. From the perspective of urban comprehensive management needs, it is proposed that the ideal platform should consist of a city management sub-platform responsible for the necessary administrative permits, a city operation sub-platform responsible for information processing, and a city service sub-platform that serves citizens, with comprehensive information and rights. Features such as clear responsibilities, functional linkage, and real-time active monitoring serve the maintenance of urban basic functions and public space management. It is proposed that the guarantee mechanism should be straightened out, the rights and responsibilities between various departments should be clarified, and various resource data should be incorporated on the basis of the spatial data base map to activate and integrate three sub-platforms of urban management, operation and service to support urban management decision-making.
\end{abstract}

\section{Introduction}

With the rapid increase of urban management affairs and the advancement of information technology, the construction of information platform related to urban management in my country is progressing rapidly. In particular, since the Ministry of Housing and Urban-Rural Development has promoted digital urban management and grid management, an integrated information platform for urban management has been initially formed in various regions, which has improved the breadth, depth and speed of urban management, and has become an "Internet + government service" system. In the construction of digital cities and smart cities, urban management has incorporated geospatial information, IoT sensors, and real-time analysis software, etc., replacing human tactics with information technology, and expanding the amount of information and availability of urban management services.

Information platform can effectively improve the efficiency and quality of urban management, which has become a consensus in the field of urban management. However, at present, there are still many problems in the large number, various types, and uneven quality of information platforms related to urban management. First of all, despite the platform has considered coordination issues, but the professional departments involved are complicated. The communication and coordination mechanism between the various departments in the platform is still not smooth, and the rights and responsibilities are not clear, which restricts the role of the platform. Secondly, the city management platform and the public security, economic information and statistics Platforms of other departments are not well connected, and there is insufficient information sharing and different data standards between platforms; thirdly, information collection is still mainly in the form of telephone complaints, urban management law enforcement and government hotlines for audio and video reporting, and IoT sensing The popularity is low, the real-time monitoring function is weak, the active monitoring function is not strong, and the real-time update level is not high.

\section{City integrated management and its information platform}

\subsection{Related concepts of urban comprehensive management}

It is generally believed that urban management is complex, its scope is difficult to define, and its content is allencompassing. The priorities of urban management at different stages of development are different. Therefore, most people tend to raise the level of awareness to the highest stage, and understand urban management as an understanding of everything in the city. The framework of the urban integrated management service platform

e-mail: CJ0840@succ.edu.cn 
explores a high degree of complexity, system and integrity. However, this understanding cannot be implemented in the administrative management of departmental responsibility and hierarchical implementation. This is also the bottleneck encountered in current urban management. The result is that strict delineation of departmental responsibility boundaries loses coordination, the establishment of comprehensive departments at the expense of efficiency, and most high-level coordination mechanisms are shelved.

Therefore, it is necessary to simplify the urban operation system. If urban development is compared to setting up a stage and performing a play, one type of department is responsible for the setting up of a stage, and another department is responsible for organizing and performing the performance. There are many different types of play to organize, but the stage is similar, and the method of setting is similar. According to this idea, the city management department can be divided into a comprehensive management department for setting up a stage and a special management department for organizing and singing. To build a platform for urban development, China has fresh practical experience. The development of Shenzhen from a fishing village into a megacity with a population of more than 10 million began with the "three connections and one levelling". Analysis believes that the various spaces enclosed by roads and underground pipelines laid along the roads, public service facilities, and building structures provide strong support for the economic and social development of the city. Among them, roads, underground pipelines and public service facilities laid along the roads support the basic functions of the city, which are necessary for the normal operation of each city. The various types of spaces enclosed by building structures can be divided into two categories. One is the responsibility management space of the owner (or user) of the building structure attached to the land (born due to planning permission, due to three guarantees in front of the door) And with), the other category is those spaces that are not allocated to individuals or units and can be used by anyone, including roads, squares, parks, etc. The latter can be understood as urban public spaces, and the division of these spaces in each city is also similar. .

\subsection{The main content of the platform}

The urban integrated management service platform is a key tool and an important tool for urban integrated management, which runs through the various links of perception, analysis, service, command and supervision in urban management. The main content of the information platform covers the basic scope of urban comprehensive management, including normal operation management and emergency incident handling, involving urban road transportation, urban water supply, drainage and sewage treatment, urban waste collection and transportation, urban energy and heat supply, urban post and telecommunications Communication and urban landscaping are mutually independent and interrelated subsystems. The platform collects, analyses, predicts, and warns of complex urban operation information, and quickly handles existing or potential urban problems: In normalized operation management, each professional subsystem generally operates independently, and different departments are responsible corresponding parts within the responsibilities and the parts that affect each other are shared in real time; in an emergency state, multiple departments participate in joint participation according to the plan to jointly deal with emergencies.

\section{3 platform frame design}

The urban integrated management service platform is the hub of urban integrated management. The core is to discover and solve problems in a timely manner. It can gradually establish an operating mechanism with fast communication, timely disposal and efficient operation among relevant departments of urban management, public service enterprises, and citizens. The ideal platform should consist of a city management sub-platform, a city operation sub-platform, and a city service sub-platform. The city management sub-platform is mainly responsible for necessary administrative permits, handling complaints, handling emergencies, and supervision and evaluation of case handling. The platform involves multiple departments, multiple links, and multiple levels. There are multiple departments such as planning bureaus and construction bureaus, multiple links such as administrative licensing, supervision, and punishment, and multiple levels such as city government, district and county government, and town (office). All departments and levels should have clear rights and responsibilities, and on the premise of ensuring that the rights and responsibilities of the departments are clear, strengthen the functional linkage between professional departments, clarify the coordination responsibilities between departments and levels, and stipulate resource sharing, information communication, and official affairs Assistance and other matters, jointly deal with urban management affairs. 


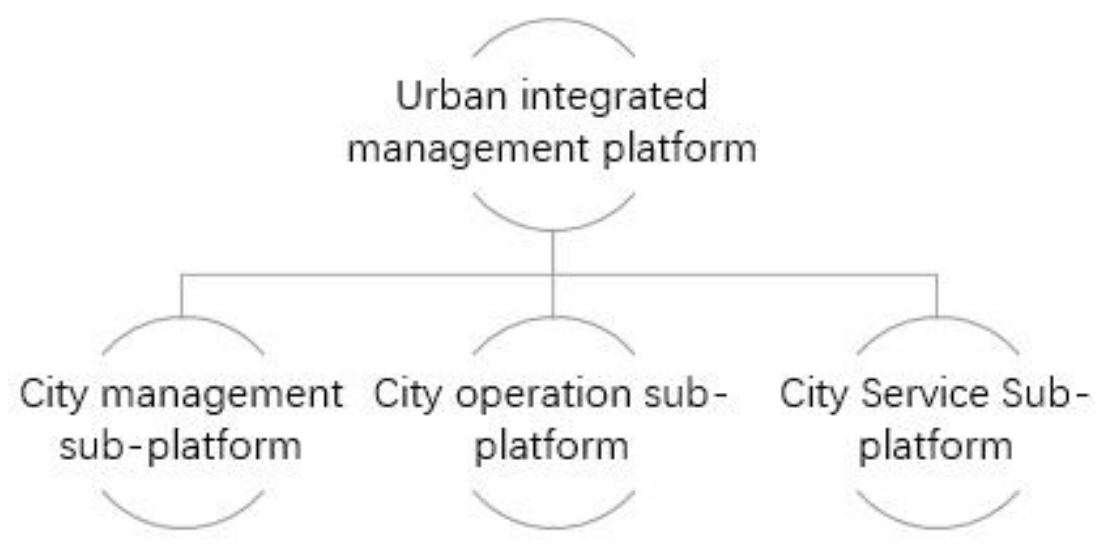

Fig 1. Architecture design of urban comprehensive management service platform

\section{Basic ideas for building a comprehensive urban management service platform}

The construction of a comprehensive urban management service platform is a systematic work. It is necessary to understand the real needs of relevant departments of comprehensive urban management, and on the basis of existing work, carefully analyse the feasibility of theoretical realization, and comprehensively design and build the implementation of the information platform path. The urban integrated management service platform cannot solve all the problems of urban development. It is necessary to do something and not to do something. The key points should be cut in, unified and separated, and the overall efficiency of the city should be improved.

Firstly, we should straighten out the system and mechanism, establish a high-level coordination organization, and form a stable platform operating procedure. Under the goal of urban healthy operation, the rights and responsibilities between different departments are clarified, and it is recommended to establish a highlevel coordination agency through joint meetings or city management committees to construct reasonable platform operating procedures to ensure the "compliance" and "division" of the operating mechanism. The so-called "combination" means to ensure the daily linkage between professional departments and specialized departments of urban comprehensive management to achieve functional complementarity and information sharing. The so-called "division" means to clarify the management and service responsibilities of different departments and optimize the separation of supervision system. The Shanghai Municipal Commission of Housing and Urban-rural Development has established a data alliance. The data and information can be exchanged among institutions within the alliance. It is difficult to ensure communication with institutions outside the alliance and cannot fully meet the requirements of urban comprehensive management; some cities in Guangdong, Zhejiang and other provinces have established big data bureaus, Coordinating all the data of the city as a whole, is responsible for collecting demand and transhipment data, and is exploring a path to establish a new coordinating agency. Through the high-level coordination agency, the platform can achieve the mechanism of the relevant departments to obtain the needs and timely share the necessary information. In terms of information security, set permissions for different departments to share and use data to ensure the security of all data.

Secondly, seek technological breakthroughs, build a spatial information database, and gradually import relevant departmental data. Taking urban planning spatial data and the on-going collection of ground and underground current situation data as a breakthrough point, we will explore two ways of real-time update database construction, including gradual update and one-time completion. In the gradual update method, complete spatial information is gradually added to the newly built area, and as the update of each section is completed, an accurate and complete multi-dimensional base map is finally formed; in the one-time completion method, for the urban area, develop a comprehensive survey of the status quo space, complete the base map at one time. During the operation of the platform, the physical information after the planning, construction, and acceptance should be incorporated into the platform in time as the platform's basic data, and the space base map should be updated in real time. Gradually incorporate the platform information and other socio-economic data of various departments, including urban residents' information, building information, infrastructure information, socio-economic data, etc., to form a standardized data support system. These professional management platforms and specialized management platforms can provide a large amount of useful information for the construction of urban integrated management information platform, and it is necessary to unify the data standards and platform architecture of each platform.

Thirdly, an efficient city management sub-platform, city operation sub-platform and city service sub-platform are formed to ensure the city's planning management, daily operations and emergency handling. The city management sub-platform executes the necessary administrative licenses to monitor, dispatch, dispose, and evaluate the city operation. Through different levels of "big linkage" platforms, we will uniformly accept information such as supervisor inspections and citizen complaints, assign corresponding subsystems according to business processes, and dispatch specific departments 
or commissioners to handle them, so as to improve the ability to find and solve problems. Through the platform, comprehensive urban management work is incorporated into the evaluation system of economic and social development and the performance evaluation of leading cadres, and various departments are promoted to perform their duties. The urban operation sub-platform focuses on infrastructure and public service facilities, and conducts data collection, analysis and early warning around urban infrastructure and public service facilities. Based on historical data, current status data, and forecast data, implement monitoring of the operation of various public spaces in the city, monitor the implementation of planning, and collect daily operational problems. The city service sub-platform serves citizens, conducts exhibitions, complaints and participation, provides inquiry services for information required by residents, as well as online handling functions for various matters, to meet the needs of residents.

\section{Conclusions}

This paper analyses the main functions that the information platform should undertake from the perspective of urban integrated management needs, and discusses the feasible implementation ideas of the future urban integrated management service platform based on the existing foundation. Undoubtedly, information will be the inevitable trend of my country's urban management development in the future, and an important means to solve the problems of serious division between departments and difficulties in linking between platforms. This paper proposes that the construction of a comprehensive urban management service platform in the future should first straighten out the operating mechanism, clarify the rights and responsibilities between various departments, and then build a spatial information base, incorporate various types of information, and on this basis, maintain the city management, operation, and operation in real time. The service sub-platform will ultimately improve the accuracy, breadth and efficiency of urban comprehensive management. An ideal information platform will benefit city managers and urban residents, and help realize the city's transformation from professional management to comprehensive management, from extensive management to refined management, and from traditional management to scientific management. Of course the research still stays in strategic analysis at the level, the specific rationality and operation path should be further discussed in conjunction with the practical progress of the case city.

\section{References}

1. Zhang Xiaojuan. (2015) Research on the elements, structure and model of smart city system. South China University of Technology.

2. Lv Xiaobiao, Zhou Junqing, Wang Cheng. (2018) On Digital City Impact on Urban Construction and Management Influence [J]. Modern City Research., 14: 61-64.
3. Wu Lun, Song Gang, Wu Qianghua, etc. (2019) From digital urban management to smart urban management: platform implementation and Key technology. Urban Development Research., 24: 99107.

4. Wang Lianfeng, Song Gang, Zhu Hui. (2018) Guidance based on the "five in one" urban management IoT platform Wave dispatch system. Egovernment., 17: 98-106. 